\title{
BEAM-BEAM EFFECTS IN CURVILINEAR INTERACTION REGIONS IN ISABELLE
}

\author{
M. Cornacchia and G. Parzen
}

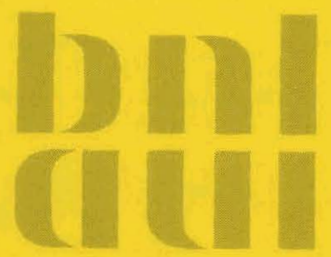

October 1979

\section{ACCELERATOR DEPARTMENT}

\section{BROOKHAVEN NATIONAL LABORATORY} ASSOCIATED UNIVERSITIES, INC. UNDER CONTRACT NO. DE-AC02-76CHOOO16 WITH THE UNITED STATES DEPARTMENT OF ENERGY 


\section{DISCLAIMER}

This report was prepared as an account of work sponsored by an agency of the United States Government. Neither the United States Government nor any agency Thereof, nor any of their employees, makes any warranty, express or implied, or assumes any legal liability or responsibility for the accuracy, completeness, or usefulness of any information, apparatus, product, or process disclosed, or represents that its use would not infringe privately owned rights. Reference herein to any specific commercial product, process, or service by trade name, trademark, manufacturer, or otherwise does not necessarily constitute or imply its endorsement, recommendation, or favoring by the United States Government or any agency thereof. The views and opinions of authors expressed herein do not necessarily state or reflect those of the United States Government or any agency thereof. 


\section{DISCLAIMER}

Portions of this document may be illegible in electronic image products. Images are produced from the best available original document. 
BNL 51103

UC-28

(Particle Accelerators and

High-Voltage Machines - TID 4500)

ISA 79-3

\section{BEAM-BEAM EFFECTS IN CURVILINEAR INTERACTION REGIONS IN ISABELLE}

M. Cornacchia and G. Parzen

October 1979

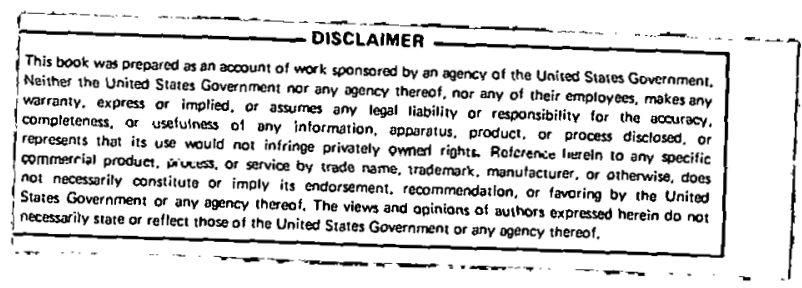

ACCELERATOR DEPARTMENT

BROOKHAVEN NATIONAL LABORATORY

ASSOCIATED UNIVERSITIES, INC.

UPTON, NEW YORK 11973

UNDER CONTRACT NO. DE-AC02-76CH00016 WITH THE

UNITED STATES DEPARTMENT OF ENERGY

DISTRIBUTION OF THIS DOCUMENT IS UNLIMITED 


\section{DISCLAIMER}

This honk was prepared as an account of work sponsored by an agency of the United States Government. Neither the United States Government nor any agency thereof; nor any of their employees, makes any warranty, expı ess u inuplicd, or assumcs any legal liability or responsibility for the accuracy, completeness, or usefulness of any information, apparatus, product, or process dıscelossed, or representiss cliat is use wuuld not infringe privately owned rights. Reference herein to any specific commercial product, process, or service by trade name, trademark, manufacturer, or otherwise, does not necessarily constitute or imply its endorsement, recommendation, or favoring by

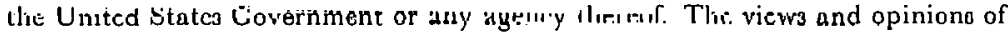
authors expressed herein do not necessarily state or reflect those of the United States Government or any agency thereof.

Printed in the United States of America

Available from

National Technical Information Service

U.S. Department of Commerce

5285 Port Royal Road

Springfield, VA 22161

Price: Printed Copy $\$ 0 ;$ Microfiche $\$ \$ 00$ 


\begin{abstract}
The presence of experimental apparatus in the interaction regions of an intersecting beam accelerator changes the configuration of the crossing beams. This changes the space-charge forces with respect to the standard, magnetfree crossing. There is strong interest in instrumenting one of the intersections at ISABELLE with a major spectrometer facility, which involves a large aperture dipole magnet located at the intersection of the beams. Various configurations of the dipole spectrometer have been proposed, and they are analyzed in this paper from the point of view of the perturbation introduced to the beam-beam interaction.
\end{abstract}




\section{INTRODUCTION}

The presence of experimental apparatus in the interaction regions of an intersecting beam accelerator changes the configuration of the crossing beams. This changes the space-charge forces with respect to the standard, magnet-free crossing. In ISABELLE, the standard collision is for the two beams to cross at an angle of $11.188 \mathrm{mrad}$ in the horizontal plane (Figure 1). There is strong interest in instrumenting one of the intersections at ISABELLE with a major spectrometer facility, which involves a largeaperture dipole magnet located at the intersection of the beams. Various configurations of the dipole spectrometer have been proposed, and we have analyzed them from the point of view of the perturbation introduced to the beam-beam interaction. The "perturbation" is defined by the extent to which the linear beam-beam tune shift departs from the standard case and by the integrated strength of the higher-order derivatives of the field. This report is concerned with the variation of the beam-beam tune shift (at one point in the ring) with respect to the standard value, on the ground that such variation will reduce the periodicity of the space-charge field and thus decrease the spacing between the resonances that these space-charge fields will excite.

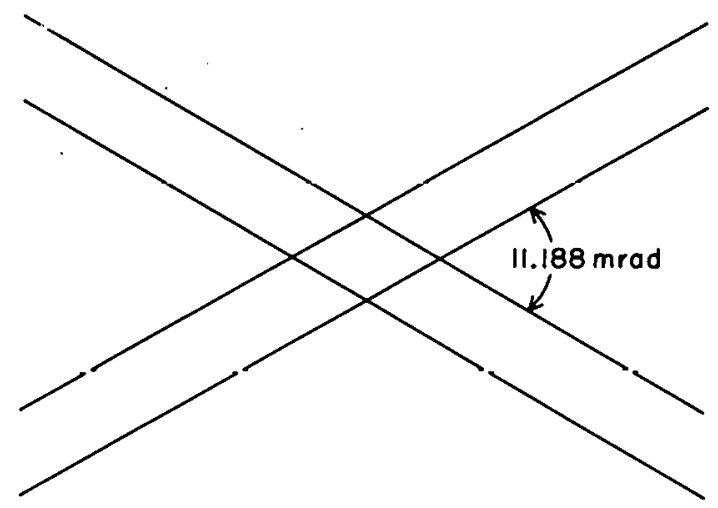

Figure 1 
The result of the analysis given below is that the vertical bending configuration (Figure 2) is the most acceptable of all the configurations examined.

\section{VERTICAL BENDING OF THE BEAM}

The proposed layout ${ }^{1}$ for this configuration is shown in Figure 2. The two beams are deflected vertically by the magnets $A$ and brought to the same height in the interaction region by the magnets $B$. Figure 2a shows the vertical projection of the beam paths; in the horizontal plane (Figure 2b), the beams cross with the standard crossing angle of $11.188 \mathrm{mrad}$. The magnet $\mathrm{C}$ has a dipole field of effective length $L_{d}$ and strength $B_{d}$. We are concerned here with the effect of the curved trajectories in $C$ on the space-charge forces acting reciprocally on the beams. Two possiblillies lave beell propused I ful hie cential magnet $c$ : a system with a 30-kG field and 4.5-m effective magnetic length and a magnet with half the strength and twice the length.
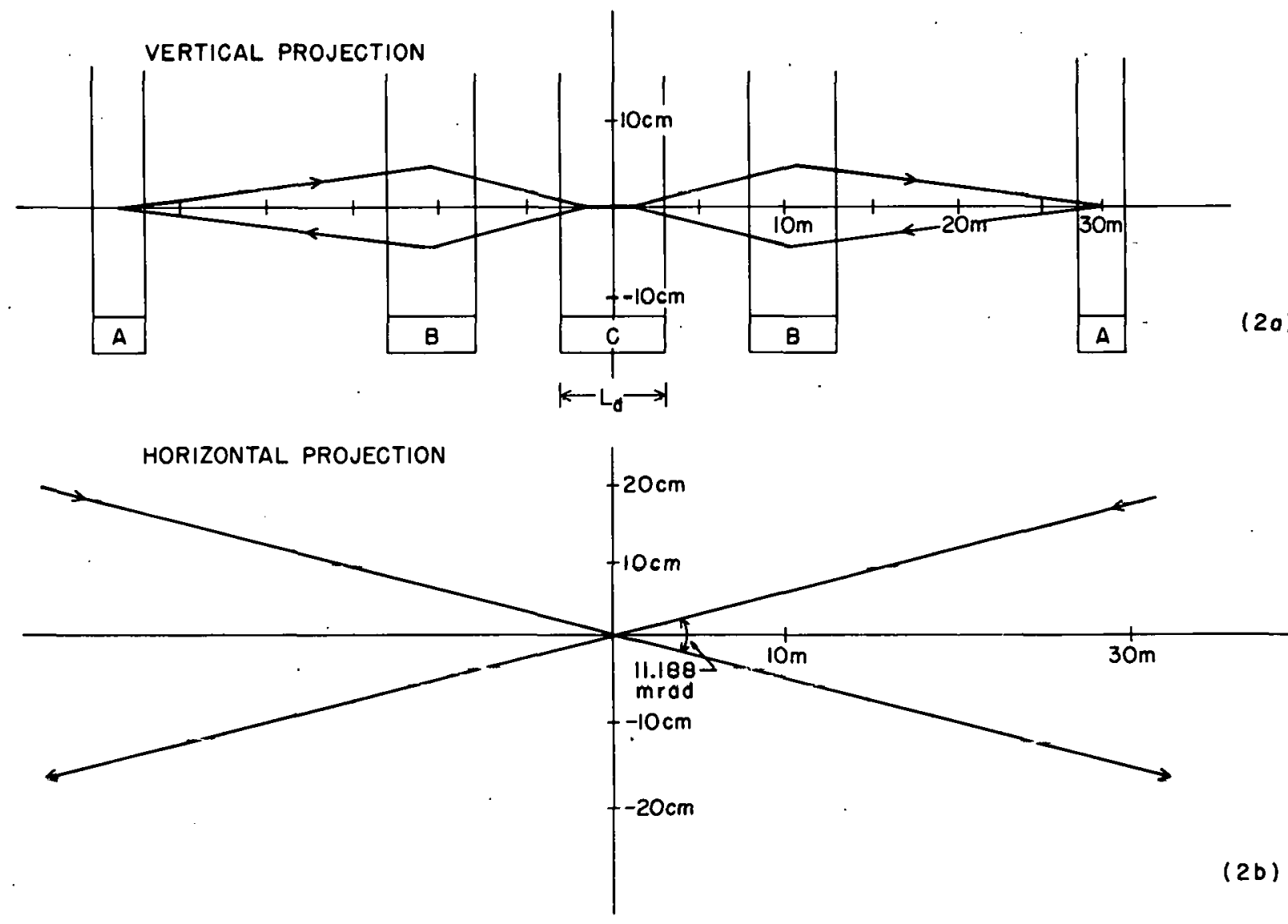

Figure 2 
A. Absence of Horizontal and Coupling Resonances

In Figure 3, the primed coordinates refer to the strong beam. In the small-angle approximation, the coordinates of the strong beam are related to those of the weak beam by the relationship

$$
\begin{aligned}
x^{\circ} & =x+s \alpha, \\
y^{\circ} & =y+\frac{s^{2}}{\rho} \\
s^{*} & =s+\frac{y s}{\rho}+x \alpha \sim s, \\
E_{x^{\prime}} & =\sim E_{x}
\end{aligned}
$$

where $\alpha$ is the horizontal crossing angle and $\rho$ is the radius of curvature in the magnet $C$. $E_{x} x$, is the space-charge field in the ultrarelativistic approximation.

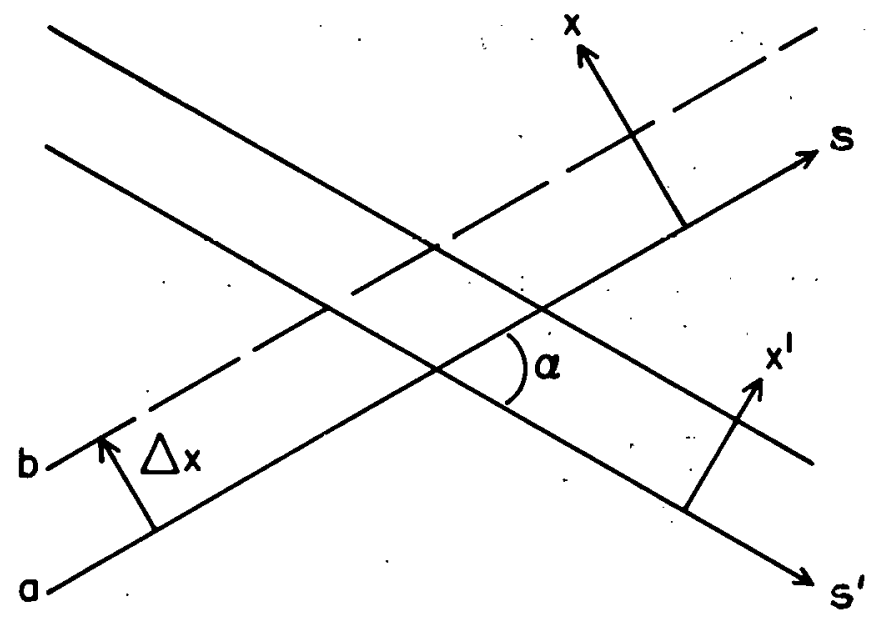

HORIZONTAL PROJECTION.

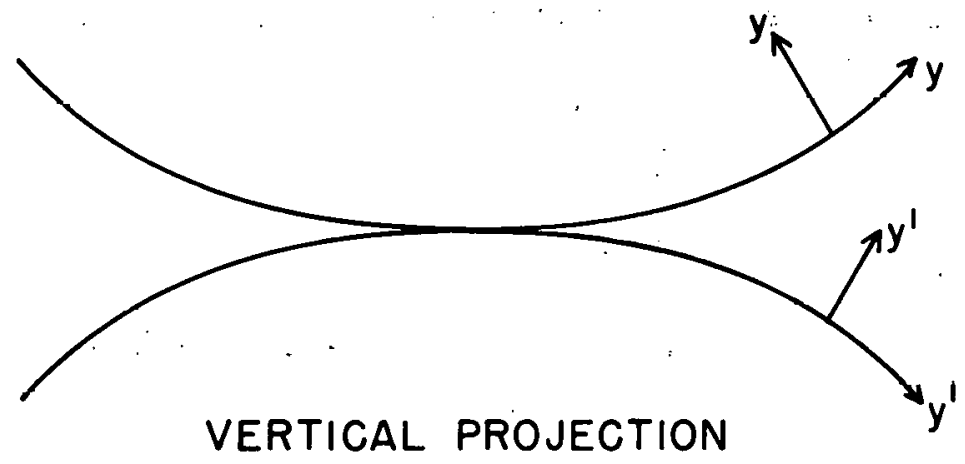

Figure 3 
Let us consider, in Figure 3, two trajectories, a and b, having the same coordinate $y(s)$, but displaced by $\Delta x$ in the coordinate system of the weak beam. If $x_{0}$ is the $x$-coordinate of trajectory $a$, we have

$$
\int_{-\infty}^{\infty} E_{x}\left[x_{0}, y(s), s\right] d s=\int_{-\infty}^{\infty} E_{x}\left[x_{0}+\Delta x, y(s), s\right] d s .
$$

We now develop $E_{x}\left[x_{0}+\Delta x, y(s), s\right]$ in a power series around $x_{0}$ :

$$
\begin{aligned}
& E_{x}\left[x_{0}+\Delta x, y(s) ; s\right]=E\left[x_{0}, y(s), s\right]+ \\
& {\left[\frac{\partial}{\partial x} E_{x}\left(x_{0}, y, s\right)\right]_{\begin{array}{c}
x \\
y
\end{array}=y(s)} \Delta x+\ldots}
\end{aligned}
$$

Since Eq. (2) is true for any $\Delta x$, we have

$$
\int_{-\infty}^{\infty} \frac{\partial}{\partial x} E_{x}(x, y, s) d s=0
$$

Thus, no horizontal beam-beam tune shift is present in this geometry, but also for all the terms responsible for the higher-order resonances:

$$
\int_{-\infty}^{\infty} \frac{\partial^{n} E_{x}}{\partial x^{n}} d s=0, \text { etc. }
$$

The assumption has been made that the variation of the horizuntal $\beta$-function in the interaction region can be neglected.

We can also consider the integral

$$
\int_{-\infty}^{\infty} E_{y} d s
$$

along the same trajectories $a$ and $b$; clearly

$$
\int_{-\infty}^{\infty} E_{y}\left[x_{0}, \dot{y}(s), s\right] d s=\int_{-\infty}^{\infty} E_{y}\left[x_{0}+\Delta x, y(s), s\right] d s,
$$

from which, proceeding as above, it follows that

$$
\int_{-\infty}^{\infty} \frac{\partial E}{\partial x} d s=\int_{-\infty}^{\infty} \frac{\partial E}{\partial y} d s=0
$$


This is also true for the higher mixed derivatives:

$$
\int_{-\infty}^{\infty} \frac{\partial^{m} E_{x}}{\partial x^{m-m}} d s=0
$$

Thus, in the geometry under consideration; where we have horizontal crossing and vertieal bending, the driving terms of the horizontal resonances and of the coupling resonances are zero (as in the standard case with horizontal crossing only).

Al1 the vertical resonances $\mathrm{m} \nu=\mathrm{p}$ are, however, driven, odd and even (unlike the standard case there only the even resonances are excited).

\section{B. The Vertical Beam-Beam Tune Shift}

The divergence theorem, for a Gaussian charge density distribution, gives the relationship between the electric field gradients:

$$
\frac{\partial E x}{\partial x}+\frac{\partial E y}{\partial y}=\frac{2 e \lambda}{\rho, 2 \pi \sigma_{x} \sigma_{y}} \exp \left(-\frac{(x+s \alpha)^{2}}{2 \sigma_{x}^{2}}-\frac{\left(y+s^{2} / 0\right)}{2 \sigma_{y}^{2}}\right)
$$

where $\lambda=1$ inear charge density, $\sigma_{x}, \sigma_{y}=$ rms values of the horizontal and vertical densities of the particles, assumed the same in the two beams, and the other symbols have their usual meanings.

Integration along the reference orbit of the weak beam $(\mathrm{x}=0, \mathrm{y}=0)$ yields

$$
\int_{-\infty}^{\infty} \frac{\partial \mathrm{E}}{\partial \mathrm{y}} \mathrm{ds}=\int_{-\infty}^{\infty} \frac{2 \mathrm{e} \lambda}{\varepsilon_{0} 2 \pi \sigma_{x} \cdot \sigma_{y}} \exp \left(-\frac{\alpha^{2}}{2 \sigma_{x}^{2}} s^{2}-\frac{s^{4}}{2 p^{2} \sigma_{y}^{2}}\right) d s
$$

since we proved in Section A that

$$
\int_{-\infty}^{\infty} \frac{\partial E}{\partial x} d s=0
$$

The vertical beam-beam tune shift is, in the extremely relativistic case $\beta \sim 1$,

$$
\Delta \nu_{y}=\frac{r_{p} \beta_{y}^{*} i}{\pi \sigma_{x} \sigma_{y} \gamma e c} \int_{-\infty}^{\infty} \exp \left(-\frac{\alpha^{2}}{2 \sigma_{x}^{2}} s^{2}-\frac{s^{4}}{2 p^{2} \sigma_{y}{ }^{2}}\right) d s
$$


where

$$
\begin{aligned}
& r_{p}=\text { classical proton radius, } \\
& \beta_{y}^{x}=\text { vertical } \beta \text {-function at the interaction point, } \\
& \gamma=\text { proton energy, normalized to the rest energy, and } \\
& i \quad=\text { beam current. }
\end{aligned}
$$

If we put $t=2 \sigma_{x}^{4} /\left(\alpha^{4} \rho^{2} \sigma_{y}{ }^{2}\right)$, the integral can be expressed as a series expansion. As a fraction of the standard vertical tune shift $\Delta \nu_{y o}$, which, in the small crossing angle approximation, has the expression

$$
\Delta \nu_{y o}=\frac{2 \beta_{y}^{*} \mathrm{r}_{\mathrm{p}} \mathrm{i}}{\sqrt{2 \pi} \text { ecy } \sigma_{\mathrm{y}} \alpha_{\mathrm{o}}}
$$

with $\alpha_{\Omega}=$ horizontal crossing angle $=11.188 \mathrm{mrad}$, we find

$$
\frac{\Delta \nu_{y}}{\Delta \nu_{y o}}=1-0.75 t+3.3 t^{2}-\ldots
$$

We have

$$
\begin{aligned}
\rho & =445 \mathrm{~m}, \\
\alpha & =11.188 \mathrm{mrad}, \\
\sigma_{x} & =1.79 \mathrm{~mm} \text { at } 30 \mathrm{GeV}, \\
\sigma_{x} & =0.49 \mathrm{~mm} \text { at } 400 \mathrm{GeV}, \\
\sigma_{y} & =0.77 \mathrm{~mm} \text { at } 30 \mathrm{GeV}, \\
\sigma_{y} & =0.21 \mathrm{~mm} \text { at } 400 \mathrm{GeV} .
\end{aligned}
$$

Thus

$$
\begin{aligned}
& t=1.11 \times 10^{-2} \text { at } 30 \mathrm{GeV}, \\
& t=8.3 \times 10^{-4} \text { at } 400 \mathrm{GeV}
\end{aligned}
$$

Retaining-only the first term of the expansion, we get

$$
\begin{aligned}
& \frac{\Delta \nu_{y}}{\Delta \nu_{y o}}=1-0.83 \times 10^{-2} \text { at } 30 \mathrm{GeV}, \\
& \frac{\Delta \nu_{y}}{\Delta \nu_{y o}}=1-6.2 \times 10^{-4} \text { at } 400 \mathrm{GeV} .
\end{aligned}
$$


Noting that the sagitta of the vertical bend, $y_{s}$, over the interaction region length is

$$
y_{s}=\frac{l^{2} \text { int }}{4 \rho}=\frac{4 \sigma_{x}{ }^{2}}{\alpha^{2} \rho},
$$

we find that the parameter $t$ is twice the square of the sagitta divided by the vertical beam size:

$$
t=2\left(\frac{y_{s}}{4 \sigma_{y}^{*}}\right)^{2} .
$$

Again, reducing the strength of the magnetic field in the dipole $\mathrm{C}$ quadratically decreases the perturbation of the linear beam-beam tune shift. We remark here, again, that our concern was for a variation of the space-charge force (as measured by the linear beambeam tune shift) from the standard value at one of the six interaction regions. This reduces the periodicity of the perturbation and decreases the spacing between the resonance lines excited by the beambeam interaction. As the calculations show, the effect is neglible for the case we have just considered.

\section{Vertical Resonances}

\section{Odd-Order Vertical Resonances}

The odd-order vertical resonances are excited because of the nonzero vertical displacement of the orbit in the crossing region. Odd-order vertical resonances are also driven by random orbit errors at the crossing point. It is of interest to compare the driving strength for these resonances due to the spectrometer with those due to the expected vertical orbit errors.

The vertical separation of the beams is given by $s^{2} / p$, at a distance $s$ from the center of magnet $C$. A rough estimate of the average beam separation can be made by putting $s=\sigma_{x} / \alpha$, where $\sigma_{x}$ is the stcandard deviation of the horizontal beam size at the crnssing poine $\left(\beta_{x}^{*}=40 \mathrm{~m}\right)$.

We have

$$
\begin{aligned}
\sigma_{\mathbf{x}} & =1.79 \mathrm{~mm} \text { at } 30 \mathrm{GeV}, \\
\sigma_{\mathbf{x}} & =0.49 \mathrm{~mm} \text { at } 400 \mathrm{GeV}, \\
\alpha & =11.188 \mathrm{mrad} .
\end{aligned}
$$

We get for the average separation,

$$
\begin{aligned}
& y_{a v}=\frac{\sigma_{x}^{2}}{\alpha^{2} \rho}=0.056 \mathrm{~mm} \text { at } 30 \mathrm{GeV}, \\
& y_{a v}=0.004 \mathrm{~mm} \text { at } 400 \mathrm{GeV},
\end{aligned}
$$


for a bending radius $\rho=445 \mathrm{~m}$, corresponding to a field $\mathrm{B}_{\mathrm{d}}=30 \mathrm{kG}$.

The same driving strength for the odd-order resonances would be obtained with a random orbit error

$$
\begin{aligned}
& y_{\text {rms }}=\frac{y_{\text {av }}}{2 \sqrt{ } 6} \text { or } \\
& y_{\text {rms }}=0.01 \mathrm{~mm} \text { at } 30 \mathrm{GeV}, \\
& y_{\text {rms }}=0.0008 \text { mm at } 400 \mathrm{GeV} .
\end{aligned}
$$

It is not known with certainty, at present, how well the random orbit errors at the crossing points can be corrected. Something like $y_{1}=0.05$ im may be a reasonable guess of what may be achieved: Thus, the driving terms for the odd-order vertical resonances due to the spectrometer magnet are probably smaller than those due to the remaining random orbit errors at the crossing polnts after these errors are corrected. The above fesults may be established more exactly. The half-width of the $N$-order resonance, $N$ being odd," due to a vertical displacement of the beams of $y=y(s)$ at one crossing point is give by ${ }^{2}$

$$
\begin{gathered}
\Delta v_{N}=\Delta v \frac{1}{2^{(N-1) / 2}} \frac{1}{[(N-1) / 2] !} \cdot A \\
A=\alpha \int_{-\infty}^{\infty} \exp \left(-x^{2} / 2 \sigma_{x}^{2}\right) \cdot y /\left(\sqrt{2 \pi} \sigma_{x} \sigma_{y}\right) \cdot d s
\end{gathered}
$$

where $\Delta \nu$ is the 1 inear vertical beam-beam tune shift and $x, y$ are evaluated along the test beam $x=\alpha s, y=y(s)$. This result is valid for beams which are Gaussian in the $x$ and $y$ directions with rms sizes $\sigma_{x}$ and $\sigma_{y}$ and for.which the path of the test beam is $\mathrm{x}=\alpha s, \mathrm{y}=\mathrm{x} y(s)$.

For the spectrometer one can evaluate the integral A using $y=s^{2} / \rho ;$ one finds

$$
A=\frac{\sigma_{x}^{2}}{\alpha^{2}\left(\rho \sigma_{y}\right)}
$$

For the orbit errors, y may be considered constant, and one finds for the rms value of A

$$
\mathrm{A}_{\mathrm{rms}}=\sqrt{6} \mathrm{y}_{\mathrm{rms}} / \sigma_{\mathrm{y}}
$$

These results give the same comparison as the previous qualitative estimate. 
It is clear that, for a magnetic field having half the strength of the one we have considered, the resonance effects are correspondingly reduced.

\section{Even-Order Vertical Resonances}

Even-order vertical resonances will also be driven as the spectrometer reduces the periodicity $f f$ the beam-beam interaction from six to one. The half-width of the $\mathrm{N}$-order resonance, $\mathrm{N}$ being even, due to a vertical displacement of beams $y(s)$ at one crossing point is given by 2

$$
\begin{aligned}
(\Delta \nu)_{N} & =\Delta \nu \frac{1}{2^{(N-2) / 2}} \frac{1}{[(N-2) / ?] !}: A \\
A & =\alpha \int_{-\infty}^{\infty} d s \frac{e^{-x^{2} / 2 \sigma_{x}^{2}}}{\left(2 \pi^{\frac{1}{2}}\right) \sigma_{x}} \cdot \frac{y^{2}}{2 \sigma_{y}^{2}}
\end{aligned}
$$

For the spectrometer one can evaluate the integral using $y=s^{2} / \rho ;$ one finds

$$
\begin{aligned}
A & =\frac{3 y_{a v^{2}}}{2 \sigma_{y}^{2}} \\
Y_{a v} & =\frac{\sigma_{x}^{2}}{\alpha^{2} p} .
\end{aligned}
$$

For the random orbit error, one finds ${ }^{2}$ the rms value of $A$

$$
A_{r m s}=\frac{\sqrt{6} \times 1.4 y_{r m s}^{2}}{2 \sigma_{y}^{2}} .
$$

Thus, the effect of the spectrometer in driving the even-order resonances is equivalent to the random orbit error with $y_{\mathrm{rms}}=\mathrm{y}_{\mathrm{av}} \frac{(3 / 3.4)^{\frac{1}{2}}}{2}$,

$$
\begin{aligned}
& y_{\text {rms }}=0.021 \mathrm{~mm} \text { at } 30 \mathrm{GeV}, \\
& y_{r m s}=0.0058 \mathrm{~mm} \text { at } 400 \mathrm{GeV} .
\end{aligned}
$$


The driving term for the even-order resonances due to the spectrometer is about the same as that due to the remaining random orbit errors at the crossing point after these orbit errors have been corrected.

The even-order resonances can also be driven by random errors in $\beta_{y}$ at the crossing points. It has been found that this drives the even-order resonances with about the same strength as that due to random errors in the orbit. Thus, the spectrometer will excite the even-order resonances with about the same strength as that due to random errors in $\beta_{y^{\circ}}$.

\section{HORIZONTAL BENDING}

A. Effect of Horizontal Bending in the Crossing Region on the BeamBeam Tune Shift.

Figures 4, 5, and 6 show other possible configurations of the dipole spectrometer. ${ }^{1}$ All the bending occurs in the horizontal plane. We consider here the effect of curved trajectories in the interaction region, defined by magnet $C$ in the figures. The $\nu$-shift introduced in this region will turn out to be small compared with the $\nu$-shift introduced in the drift space between magnets $B$ and $C$, which is computed later in this section. The beam separation $\Delta x$ is given by

$$
\Delta \mathbf{x}=\alpha s-\frac{s^{2}}{\rho}
$$

where $s$, the longitudinal distance, is measured from the center of magnet $\mathrm{C}$, and $\alpha$ is the crossing angle at the center of magnet $C$.

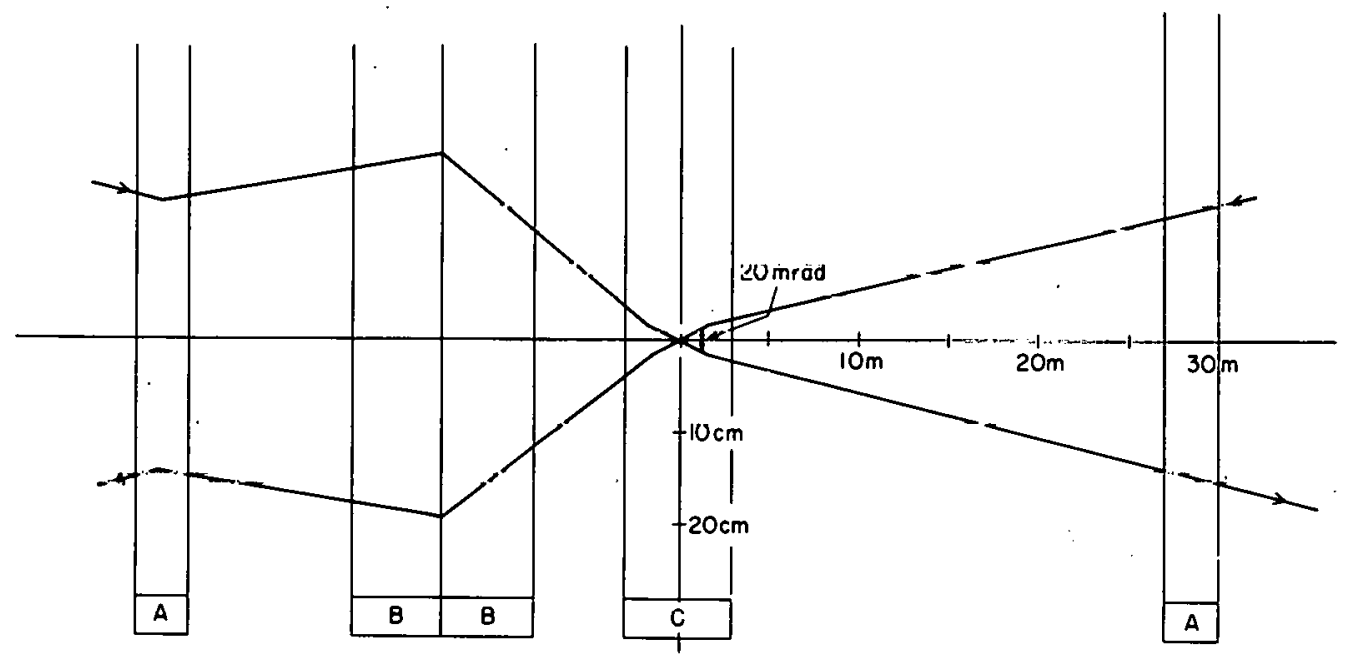

Figure 4 


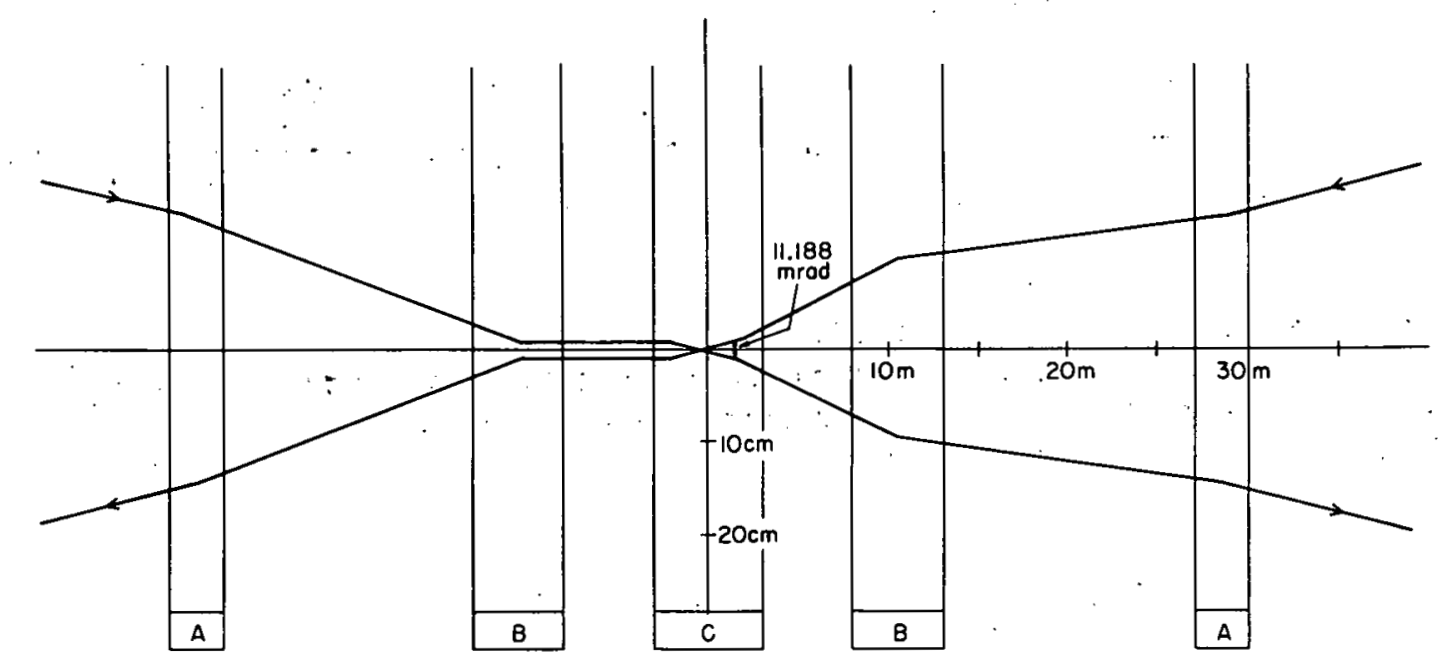

Figure 5

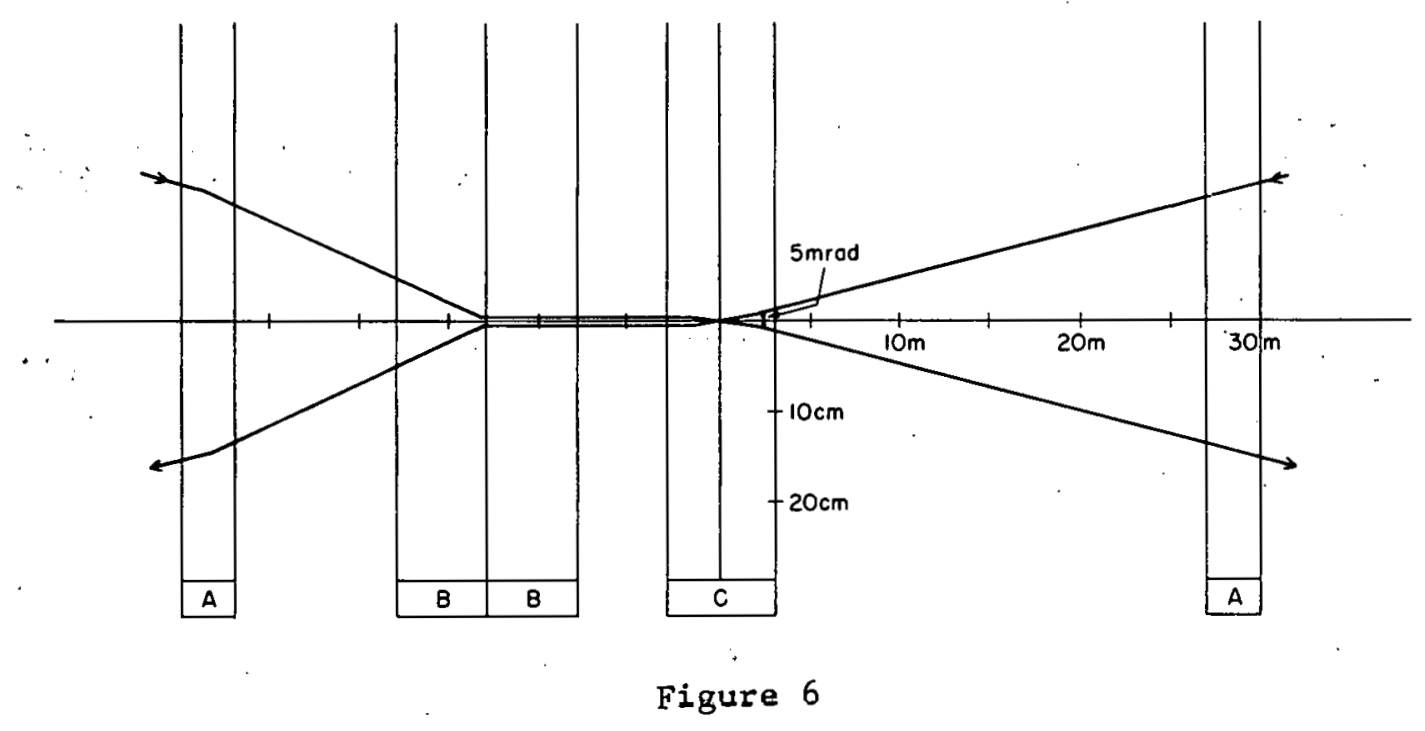

It is clear that, in case of curved trajectories, the assumption

$$
\int_{-\infty}^{\infty} \frac{\partial E}{\partial x} d s=0
$$

1s no longer valid. Hence, a horizontal tune shift will result. Since we expect this to be sma1l, we avoid solving for the field, which, for a beam that is not round, requires a rather cumbersome calculation, 3 and are satisfied with establishing the order " of magnitude of the perturbation. 
The divergence theorem gives

$$
\int_{-\infty}^{\infty} \frac{\partial E_{x}}{\partial x} d s+\int_{-\infty}^{\infty} \frac{\partial E_{y}}{\partial y} d s=\frac{2 e \lambda}{e_{0}^{2 \pi \sigma_{x} \sigma_{y}}} \int_{-\infty}^{\infty} \exp \left[-\frac{\left(\frac{s^{2}}{\rho}-s \alpha\right)^{2}}{2 \sigma_{x}^{2}}\right] d s
$$

where the integration is performed along the trajectory $x=x(s), y=0$. Since the beams are we11 separated at the entrance and exit of magnet $c$, we may assume that al1 the interaction takes place within the magnetic field region. After integration, we find

$$
\Delta v_{x}\left(\frac{e_{y}^{*}}{\beta_{\dot{x}}^{*}}\right)+\Delta v_{y}=\Delta v_{y o}\left[1+3 t-\frac{15}{2} t^{2}+\ldots\right]
$$

where $\Delta \nu_{\text {po }}$ is the vertical beam-beam tune shift for standard crossing, $B^{*}$ and $B^{\prime 0}$ are the horizontal and vertical $\beta$-functions at the crossing pồint, and

$$
t=\frac{2 \sigma_{x}^{2}}{\alpha^{4} \rho^{2}}
$$

is a measure of the perturbation introduced by the curved trajectories. Since $t \ll 1$, we may consider only the first term of the solution (31), which gives

$$
\frac{\Delta \tilde{\nu}_{x, y}}{\Delta \nu_{\text {yo }}}=\frac{\Delta \nu_{y}-\Delta \nu_{y o}}{\Delta \nu_{y o}}+\frac{\Delta \nu_{x}\left(\beta_{y}^{*} / \beta_{x}^{*}\right)}{\Delta \nu_{y o}} \approx 6,0 \times 10^{-4}
$$

for the layout of Figure 4, where

$$
\alpha=20 \mathrm{mrad}, \rho: 445 \mathrm{~m} .
$$

For the layout of Figure 5, where $\alpha=11.188 \mathrm{mrad}, \rho=445 \mathrm{~m}$,

$$
\frac{\Delta \tilde{v}_{x, y}}{\Delta v_{\text {yo }}} \approx 6.2 \times 10^{-3}
$$

and, finally, for the layout of Figure 6 , where $\alpha=5$ mrat, $\beta=890 \mathrm{~m}$,

$$
\frac{\Delta \tilde{\nu}_{\mathrm{x}, \mathrm{Y}}}{\Delta \nu_{\mathrm{yo}}} \approx 3.9 \times 10^{-2}
$$

It is clear that in the cases shown in Figures 4 and 6 the effect of curved trajectories in the interaction region is neglibilble 
compared with the variation of the crossing angle with respect to the standard value ( 11.188 mrad). In the case of Figure 5, the effect of curved trajectories is still small compared with the shift introduced by the interaction of the beams in the drift space between magnets $C$ and $B$, as shown below.

\section{B. Effect of "Paralle1" Beams}

In the layouts of Figures 5 and 6 the beam paths lie close to each other for a considerable distance. Since in both cases the beams are not exactly parallel, we write for the separation $\Delta x$ in the field-free region

$$
\Delta \mathrm{x}=\Delta \mathrm{x}_{\mathrm{o}}+\mathrm{K}\left(\mathrm{s}-\mathrm{s}_{\mathrm{o}}\right)
$$

This beam separation in the drift space between magnets $C$ and $B$. $(\sim \mathrm{m})$ varies, from right to left,

$$
\begin{aligned}
& \Delta \mathrm{x}_{\mathrm{o}}=13.8 \mathrm{~mm} \text { to } \Delta \mathrm{x}_{1}=19.2 \mathrm{~mm} \text {, in Figure } 5, \\
& \Delta \mathrm{x}_{\mathrm{o}}=5.6 \mathrm{~mm} \text { to } \Delta \mathrm{x}_{1}=5.3 \mathrm{~mm} \text {, in Figure } 6
\end{aligned}
$$

The longitudinal distance $s$ is measured from the crossing point (center of magnet $\mathrm{C}$ ); $\mathrm{s}_{\mathrm{g}}$ is the distance of the left edge of the magnet $C$ from the crossing point.

Since the beam separation is many times its rms beam size, it is a reasonable approximation to assume round beams, which greatly simplifies the problem. We have

$$
\left(\frac{\partial \mathrm{E}_{\mathrm{x}}}{\partial \mathrm{x}}\right)_{\substack{\mathrm{x}=\Delta \mathrm{x} \\ \mathrm{y}=0}} \approx-\left(\frac{\partial \mathrm{E} y}{\partial \mathrm{y}}\right)_{\substack{\mathrm{x}=\Delta \mathrm{x} \\ \mathrm{y}=0}}=\frac{\lambda \mathrm{e}}{\pi \varepsilon_{0} \Delta \mathrm{x}^{2}} \text {. }
$$

The separation $\Delta \mathrm{x}$ is given by Eq. (37). In calculating the tune shift, we must also take into account the variation of the $\beta$-function along the trajectory:

$$
\beta_{x, y}(s)=\beta_{x, y}^{*}+\frac{s^{2}}{\beta_{x, y}^{*}} .
$$

We have, integrating over the field-free region $s_{0}-s_{1}$

$$
\Delta v_{x, y}=\frac{r_{p} i}{\pi y c e} \int_{s_{0}}^{s_{1}} \frac{1}{\left[\Delta x_{0}+k\left(s-s_{0}\right)\right]^{2}}\left(\beta_{x, y}^{*}+\frac{s^{2}}{\beta_{x, y}^{*}}\right) d s
$$


After integration, we find for the ratios between the tune shift and the vertical tune shift for standard crossing $\Delta \nu_{\text {yo }}$,

$$
\begin{aligned}
& \frac{\Delta \nu_{x}}{\Delta \nu_{\text {yo }}}=0.35 ; \quad \frac{\Delta \nu_{y}}{\Delta \nu_{y o}}=0.10 \text { at } 30 \mathrm{GeV}, \\
& \frac{\Delta \nu_{x}}{\Delta \nu_{\text {yo }}}=0.10 ; \quad \frac{\Delta \nu_{y}}{\Delta \nu_{y o}}=0.027 \text { at } 400 \mathrm{GeV},
\end{aligned}
$$
$f_{q^{r}}$ the layout of Figure 5 and with the standard values $\beta_{x}^{*}:=40 \mathrm{~m}$,
$\beta_{y}^{*}=7.9 \mathrm{~m}$.

The layout of Figure 6 gives, with the same $R_{x}^{*}, R_{y}^{*}$,

$$
\begin{aligned}
& \frac{\Delta \nu_{x}}{\Delta \nu_{\text {yo }}}=3.11 ; \quad \frac{\Delta \nu_{y}}{\Delta \nu_{\text {yo }}}=0.90 \text { at } 30 \mathrm{GeV}, \\
& \frac{\Delta \nu_{x}}{\Delta \nu_{\text {yo }}}=0.85 ; \quad \frac{\Delta \nu_{y}}{\Delta \nu_{\text {yo }}}=0.24 \text { at } 400 \mathrm{Gev} .
\end{aligned}
$$

Note the very large horizontal tune shift. Since $\Delta v_{y}=-0.0064$ at $30 \mathrm{GeV}, \Delta \nu_{x}=-0.02$ for the configuration of Figure 6 ?

Inside magnet $B$ of length $t_{B}$ (Flgures 5 and 6 ), the separation between the two beams is

$$
\Delta x=\frac{\left(s-s_{2}\right)^{2}}{p}+\alpha\left(s-s_{2}\right)+\Delta x_{u}
$$

with

$$
\alpha=1.08 \text { mrad, } p=740 \mathrm{~m}, s_{2}-7.25 \mathrm{~m}, b_{B} \Rightarrow 5 \mathrm{~m}, \Delta x_{0}-19.2 \text { um }
$$
(Figure 5).

and

$$
\begin{aligned}
& \alpha=-0.06 \mathrm{mrad}, \rho=2322 \mathrm{~m}, \mathrm{~s}_{2}=7.25 \mathrm{~m}, l_{B}=10 \mathrm{~m}, \Delta \mathrm{x}_{\mathrm{o}}=5.3 \mathrm{~mm} \\
& \text { gure 6). }
\end{aligned}
$$
(Figure 6). 
The beam-beam tune shift is, in this case,

$$
\Delta \nu_{x, y}=\frac{r_{p} i}{\pi y c e} \int_{0}^{l_{B}} \frac{1}{\left(\Delta x_{0}+\alpha s+\frac{s^{2}}{\rho}\right)^{2}}\left(\beta_{x, y}^{*}+\frac{\left(s+s_{2}\right)^{2}}{\beta_{x, y}^{*}}\right) d s
$$

After integration we find for the configuration of Figure 5:

$$
\begin{aligned}
& \frac{\Delta \nu_{x}}{\Delta \nu_{\text {yo }}}=0.06 ; \quad \frac{\Delta \nu_{y}}{\Delta \nu_{y o}}=0.009 \text { at } 30 \mathrm{GeV}, \\
& \frac{\Delta \nu_{x}}{\Delta \nu_{\text {yo }}}=0.016 ; \frac{\Delta \nu_{y}}{\Delta \nu_{y o}}=0.002 \text { at } 400 \mathrm{GeV} ;
\end{aligned}
$$

and for the configuration of Figure 6

$$
\begin{aligned}
& \frac{\Delta \nu_{x}}{\Delta \nu_{y_{0}}}=1.47 ; \quad \frac{\Delta \nu_{y}}{\Delta \nu_{y_{0}}}=0.86 \text { at } 30 \mathrm{GeV}, \\
& \frac{\Delta \nu_{x}}{\Delta \nu_{\text {yo }}}=0.40 ; \quad \frac{\Delta \nu_{y}}{\Delta \nu_{\text {yo }}}=0.23 \text { at } 400 \mathrm{GeV} .
\end{aligned}
$$

As far as the resonance excitation is concerned, the "quasiparallel" beam layouts of Figures 5 and 6 will also excite the pure horizontal resonance, $n \nu_{x}=P$, as well as all the coupling resonances, $\mathrm{n} \nu_{\mathrm{x}}+\mathrm{m} \nu_{\mathrm{y}}=\mathrm{P}$, for which $\mathrm{m}$ is even. These are the usual resonances present In a field with median plane symmetry. There is no special symmetry here that would cause the horizontal and coupling resonances to be absent, as in the case when the spectrometer field io romoved.

\section{CONCLUSIONS}

We have analyzed, from the point of view of the beam-beam interaction, the effect of magnetic fields of different geometry in the interaction region of the crossing beams in ISABELLE. The assumption was made that a linear beam-beam tune shift which, at only one of the six interaction points, is appreciably different from all the rest, will reduce from 6 to 1 the periodicity of all the resonances excited by the space-charge force and therefore is a potential source of trouble. Other effects of the presence of resonances excited by the beam-beam interaction which would not otherwise be present in standard crossing geometry have been considered. The result of the 
analysis is that the vertical bending case (Figure 2) is the most acceptable of all the cases examined, at least from the point of view of the beam-beam interaction. The variation of the linear tune shift, as compared with the standard case, is negligible; no coupling resonances are driven by the space-charge field. The strength of the odd-order resonances (not present in a standard crossing geometry), after they are corrected, falls withing the range of those excited by random vertical closed orbit errors.

The horizontal bending cases we have examined impose an obvious asymetry on the regular distribution of the space-charge forces, and excite additional horizontal and coupling resonances. They. also produce rather large linear tune shifts.

\section{REFERENCES}

1. S. Ozaki, Private communication, 1979.

2. G. Parzen, BNL ISABELLE Project Tech. Note 136, 1979.

3. B. W. Montague, Calculation of Liminosity and Beam-Beam Detuning in Coasting-Beam Interaction Regions, CERN/ISR-GS/75-36.

vm

Distribution - External 